\title{
Escala do comportamento do treinador \\ - versão treinador (ECT-T) e versão atleta (ECT-A): \\ o que o treinador diz é confirmado pelos seus atletas?
}

CDD. 20.ed. 796.011

796.073
Luiz Carlos Couto de Albuquerque MORAES*

Edson Soares MEDEIROS FILHO*

Ingrid Ludmila Bastos LÔBO**

Dêner Rodrigues da SILVEIRA***
*Universidade Federal de Minas Gerais.

**Fundação Mineira de

Educação e Cultura.

***Universidade Católi-

ca de Góias.

\section{Resumo}

Objetivou-se verificar o nivel de associação entre as percepções de atletas e treinadores de esportes coletivos e individuais nas seis dimensões do comportamento do treinador - Treinamento Físico (TF), Treinamento Técnico (TT), Preparação Mental (PM), Estabelecimento de Objetivos (E0), Reforço Pessoal Positivo (RPP), Reforço Pessoal Negativo (RPN) - acessado pela Escala do Comportamento do Treinador - Versão Treinador (ECT-T) e Versão Atleta (ECT-A). Participaram 181 atletas ( 114 homens e 67 mulheres) de nove modalidades esportivas com idade média de 17,15 anos $( \pm 1,48)$ e seus respectivos treinadores $(N=32)$, todos do sexo masculino, com idade média de 32,88 $( \pm 8,91)$. Treinadores e atletas responderam aos 38 itens de mesmo conteúdo dos questionários mencionados. A correlação não-paramétrica de Spearman mostrou a inexistência de associação significativa, ao nivel $\alpha=0,05$, entre as percepções treinadores e atletas de modalidades coletivas nas dimensões $\Pi$, PM, EO e RPP. Entre treinadores e atletas de modalidades individuais também não foram verificadas associações significativas em cinco dimensões: TF, T, PM, EO e RPP. Dentre outras razões, a baixa compatibilidade entre as percepções de atletas treinadores, pode estar relacionada a falhas no processo de organização do treinamento. Além disso, o relacionamento de cada treinador com os atletas sob o seu comando parece informar mais do que a simples categorização: modalidades coletivas versus individuais.

UnIteRmos: Comportamento do Treinador, Liderança; Psicologia do Esporte; ECT-T \& ECT-A.

\section{Introdução}

O comportamento do treinador influencia no nível de performance alcançado pelos aprendizes, conforme constataram estudos envolvendo expoentes de diferentes áreas de atuação (BLOOM, 1985; ERICSSON, Krampe \& Tesch-RÖMER, 1993; Salmela \& Moraes, 2003). Particularmente no esporte, o comportamento do treinador influencia no desenvolvimento da performance dos atletas (MEDEIROS Filho \& Moraes, 2004; Moraes, 1996; Salmela, Young \& KALLIO, 2000), haja visto que os treinadores auxiliam os seus atletas a estabelecerem objetivos e alcançarem altos níveis de preparação física, técnica, tática e psicológica (DURAND-Bush, SALMELa \& Green-Demers, 2001; Janelle \& Hillman, 2003; Moraes \& Lôbo, 2001; Orlick, 2000).
Acerca da importância dos treinadores no esporte, CHELLADURA (1990) observou que treinadorese professores de sucesso entendem as necessidades dos atletas e procuram estabelecer uma ação de correspondência entre seus comportamentos e o grau de desenvolvimento de seus atletas.

Côté, Salmela, Trudel, Baria e Russell (1995) apresentaram um modelo teórico que confirmaaimportância do comportamento do treinador. Após entrevistarem 17 treinadores canadenses considerados "experts" em ginástica olímpica, esses pesquisadores relataram em um modelo teórico, intitulado Modelo do Treinador (MT), as características centrais- organização, treinamento e competição - e periféricas - características pessoais dos treinadores, características pessoais dos atletas e fatores contextuais - que interferem na promoção da performance dos atletas. 
Além da identificação dos fatores, centrais e periféricos, que promovem a competência dos atletas, o MT possibilitou a elaboração, e posterior validação, de um instrumento (vide Cótê, Yardley, Hay, SeDWICK \& BAKER, 1999) orientando a análise do comportamento do treinador, denominado de Coaching Behavior Scale for Sport (CBS-S) ou, na tradução para a língua portuguesa brasileira, de Escala de Comportamento do Treinador, surgindo, portanto, dois instrumentos fundamentados no CBS-S e que foram recentemente validados no Brasil. A Escala de Comportamento do Treinador Versão Treinador - ECT-T (Silveira, 2005) e Versão Atleta - ECT-A (Lôbo, Moraes \& Nascimento, 2005) possibilitam uma comparação entre as percepções dos atletas (ECT-A) com a dos treinadores (ECT-T), em relação a diferentes situações que correspondem ao comportamento dos treinadores durante o treinamento.

Apesar do consenso sobre a importância do treinador no esporte (ERicsson, 2003; Moraes, Salmela, 2002; Salmela, 1996; SMITH \& SMOLl,1984), a maior parte da literatura na área da psicologia esportiva não tem considerado conjuntamente "o ponto de vista" do atleta e o "ponto de vista" do treinador em estudos sobre liderança no esporte. Noutras palavras, pesquisas que comparam o comportamento do treinador - declarado pelo próprio treinador - com a percepção que os atletas têm do comportamento do treinador são raras ou mesmo inexistentes.

Sendo assim, a utilização conjunta do ECT-T e do ECT-A permite verificar as possíveis diferenças ou similaridades envolvendo as respostas dos treinadores e de seus atletas e, consequentemente, possibilita um melhor entendimento acerca do processo de interação treinador-atleta.

O ECT-T foi utilizado para avaliar a percepção dos treinadores acerca de seus próprios comportamentos, enquanto o ECT-A mediu a percepção dos atletas em relação a comportamentos dos seus treinadores.

Portanto, este estudo tratou de verificar o nível de correspondência entre as percepções de atletas e seus respectivos treinadores em esportes coletivos e individuais. Mais especificamente, investigou-se o nível de associação entre as percepções de atletas e treinadores de esportes coletivos e individuais em seis dimensões do comportamento do treinador (Treinamento e Condicionamento Físico, Treinamento Técnico, Preparação Mental, Estabelecimento de Objetivos, Reforço Pessoal Positivo e Reforço Pessoal Negativo). Percepção no presente estudo assumiu conotação de "formar a idéia de; compreender" (vide HouaIss, ViLlar \& Franco, 2001). Noutras palavras, procuramos investigar qual a ideia/compreensão que treinadores têm do seu próprio comportamento e comparar com a ideia/compreensão que atletas têm acerca do comportamento dos seus treinadores.

Tendo em vista que, comumente, treinadores de esportes individuais treinam um menor número de atletas e, por conseguinte, desfrutam de mais tempo e possibilidades para interagirem com eles, nós hipotetizamos que: a força do coeficiente de correlação seria maior entre as percepções de atletas e treinadores de esportes individuais do que entre as percepçóes de atletas e treinadores de esportes coletivos.

\section{Materiais e métodos}

\section{Sujeitos}

Participaram do estudo 181 atletas (TABELA 1), 114 homens e 67 mulheres, de nove esportes diferentes (TAB. 1$)$ com idade variando entre 15 e 20 anos $(M=$ $17,15 ; D P=1,48)$ e seus respectivos treinadores $(\mathrm{n}=$ 32 ), todos do gênero masculino, com idade variando entre 20 e 50 anos (média $=32,88 ; \pm 8,91$ ).

Neste estudo, treinadores e atletas treinavam juntos há pelo menos um mês, a fim de que, baseados nas experiências vividas, pudessem responder a ECT-T e a ECT-A. Em média, os atletas de esportes coletivos conviviam com seus treinadores havia 4,87 meses $(\mathrm{DP}=6,37)$, enquanto os de modalidades individuais conviviam em média com seus treinadores havia 4,43 $(\mathrm{DP}=2,25)$. Conforme relato de WeInBERG e GOULD
(2001) é difícil precisar o tempo necessário para a identificação dos liderados com os seus respectivos líderes, uma vez que cada grupo composto por treinador e atletas possui características muito singulares.

Os treinadores das modalidades coletivas possuíam, em média, 9,45 anos de experiência $(D P=7,34)$ nos seus respectivos esportes; os individuais possuíam média de 14,69 anos de experiência $(D P=8,62)$. Dentre os primeiros, $42,1 \%$ tinham curso superior, $36,8 \%$ haviam concluído o ensino médio, $15,8 \%$ o ensino fundamental e 5,3\% não possuíam nenhum nível de instrução formal completo. Dentre os treinadores de esportes individuais 38,5\% tinham curso superior completo, $46,2 \%$ ensino médio completo, $7,7 \%$ ensino fundamental completo e $7,7 \%$ não possuíam nenhum nível de instrução formal completo. 
TABELA 1 - Modalidades esportivas e sexo dos atletas e treinadores participantes do estudo.

\begin{tabular}{|c|c|c|c|c|}
\hline \multirow{2}{*}{ Modalidade } & \multirow{2}{*}{ Esportes } & \multicolumn{2}{|c|}{ Atletas } & \multirow{2}{*}{$\begin{array}{c}\text { Treinadores } \\
\mathbf{M}\end{array}$} \\
\hline & & $\mathbf{M}$ & $\mathbf{F}$ & \\
\hline \multirow[t]{5}{*}{ Coletiva } & Basquetebol & 10 & 0 & 2 \\
\hline & Futebol & 30 & 0 & 1 \\
\hline & Futsal & 9 & 24 & 6 \\
\hline & Handebol & 14 & 29 & 7 \\
\hline & Voleibol & 13 & 3 & 3 \\
\hline \multicolumn{2}{|l|}{ Total } & 76 & 56 & 19 \\
\hline \multirow[t]{4}{*}{ Individual } & $\begin{array}{l}\text { Ginástica } \\
\text { artística }\end{array}$ & 2 & 4 & 1 \\
\hline & Jiu-Jitsu & 10 & 0 & 4 \\
\hline & Karatê & 20 & 7 & 7 \\
\hline & Taekwondo & 6 & 0 & 1 \\
\hline Total & & 38 & 11 & 13 \\
\hline Total geral & & \multicolumn{2}{|c|}{181} & 32 \\
\hline
\end{tabular}

\section{Instrumentos}

Utilizaram-se os instrumentos ECT-T (SIIVEIRA, 2005) e ECT-A (Lôbo, Moraes \& Nascimento, 2005) que permitem avaliar a liderança do treinador por meio da comparação entre a sua percepção com a dos atletas sob o seu comando. Destaca-se que ambos os instrumentos apresentam requisitos psicométricos que respaldam a sua utilização no contexto brasileiro: no ECT-A os valores de consistência interna variaram de 0,81 (Reforço Pessoal Negativo) a 0,92 (Estabelecimento de Objetivos) e no ECT-T de 0,70 (Reforço Pessoal Negativo) a 0,79 (Preparação Mental).

Esses questionários possuem 40 questóes cada um e abordam seis dimensões do comportamento do treinador - Treinamento e Condicionamento Físico (TF), Treinamento Técnico (TT), Preparação Mental (PM), Estabelecimento de Objetivos (EO), Reforço Pessoal Positivo (RPP), Reforço Pessoal Negativo (RPN) -, de acordo com a frequência de comportamentos específicos dos mesmos, possibilitando no ECT-T as opçōes de 1 (nunca) a 5 (sempre) e no ECT-A as opções de 1 (nunca) a 7 (sempre).

Embora cada teste possua 40 questóes, somente 38 foram comparadas porque duas não compunham o rol de perguntas de ambos os questionários, ou seja, constavam em apenas um dos dois testes. O QUADRO 1 apresenta o número de questōes comparadas em cada uma das dimensões e fornece um exemplo.

QUADRO 1 - Exemplo e número de questões comparadas em cada uma das seis dimensões dos questionários ECT-T e ECT-A (Adaptado de SilvEIRA, 2005).

\begin{tabular}{|l|l|l|}
\hline \multirow{2}{*}{ Dimensóes } & \multicolumn{1}{|c|}{ Questionários } \\
\cline { 2 - 3 } & \multicolumn{1}{|c|}{ ECT-T } & \multicolumn{1}{c|}{ ECT-A } \\
\hline TF $(\mathrm{N}=7)$ & $\begin{array}{l}\text { Forneço ao atleta um programa anual de } \\
\text { treinamento? }\end{array}$ & Fornece(m)-me um programa anual de treinamento? \\
\hline TT $(\mathrm{N}=8)$ & $\begin{array}{l}\text { Certifico-me de que o atleta esteja entendendo as } \\
\text { técnicas e as estratégias que lhe estão sendo ensinadas? }\end{array}$ & $\begin{array}{l}\text { Assegura-se de que eu estou entendendo as } \\
\text { técnicas que me estão sendo ensinadas? }\end{array}$ \\
\hline PM $(\mathrm{N}=5)$ & $\begin{array}{l}\text { Dou conselho ao atleta sobre como atuar } \\
\text { sobre pressão? }\end{array}$ & $\begin{array}{l}\text { Aconselha(m)-me sobre como desempenhar em } \\
\text { momentos de pressão? }\end{array}$ \\
\hline EO $(\mathrm{N}=5)$ & Ajudo-o a estabelecer objetivos a longo prazo? & Ajuda(m)-me a estabelecer objetivos a longo prazo? \\
\hline RPP $(\mathrm{N}=6)$ & $\begin{array}{l}\text { Sou digno de confiança em relação aos proble- } \\
\text { mas pessoais do atleta? }\end{array}$ & $\begin{array}{l}\text { É digno de confiança em relação aos meus } \\
\text { problemas pessoais? }\end{array}$ \\
\hline RPN $(\mathrm{N}=7)$ & Grito com o atleta quando estou com raiva? & Grita comigo quando está com raiva? \\
\hline
\end{tabular}

\section{Coleta de dados}

Antes do início desta fase, o presente estudo foi aprovado pelo Comitêde Ética em Pesquisa da UFMG. Treinadores e atletas foram informados sobre os objetivos, a relevância e os procedimentos metodológicos do estudo, tendo consentido voluntariamente em participar do mesmo.

A coleta de dados ocorreu nas dependências dos próprios clubes dos voluntários e durante competiçôes esportivas de âmbito estadual. Juntamente com as instruçôes relativas ao correto preenchimento dos instrumentos, os treinadores e atletas receberam um exemplar do questionário e uma caneta, para que pudessem responder às questões.

Solicitou-se aos participantes da pesquisa (treinadores e atletas) que preenchessem individualmente o questionário, evitando que suas respostas fossem influenciadas por outrem.

Puderam também despender o tempo necessário na elaboração de suas respostas. 


\section{Análise de dados}

Para cada uma das 38 questōes contrastadas, as respostas dos atletas foram sumarizadas por meio de média e, o resultado, quando um valor fracionado, foi arredondado por completo. Desse modo, nas seis dimensões analisadas, correlacionou-se respostas do treinador, nas questôes de cada um dos 38 itens, com a média da percepção dos seus atletas (também em cada questão).

\section{Resultados}

Antes de se correlacionar às respostas dos atletas e treinadores, testou-se com um nível de confiabilidade de $95 \%$ a hipótese nula de que o tempo de convivência dos atletas de esportes coletivos com os seus treinadores é igual ao tempo de convivência de atletas de desportos individuais com os seus treinadores. $\mathrm{O}$ resultado do Test- $t$ de Student para dois grupos independentes, (Teste $\mathrm{t}=1,70 ; \mathrm{GL}=962 ; \mathrm{p}=0,09)$, confirma a hipótese nula. Esse resultado permite afirmar que possíveis diferenças na compatibilidade entre os grupos formados pela díade treinadores-atletas de esportes coletivos e treinadoresatletas de esportes individuais não decorrem de diferenças no tempo de convivência entre os treinadores e atletas desses grupos.

Também comparou-se, por meio do Teste de Aderência do Qui-Quadrado, a distribuição de frequências em relação ao nível de instrução formal dos treinadores dos grupos individual e coletivo $\left(\chi^{2}\right.$ $=0,67 ; \mathrm{GL}=3 ; \mathrm{p}=0,90)$. Novamente, conforme exposto, não se pode atribuir possíveis dissimilaridades nos resultados dos coeficientes de correlação à escolaridade dos treinadores dos grupos em questão.

A TABELA 2 apresenta os resultados oriundos da análise de cada uma das seis dimensões avaliadas. Após a mesma, os resultados são brevemente descritos e na sequência pormenorizados e interpretados.

TABELA 2 - Coeficiente de correlação de Spearman para as dimensões avaliadas pela ECT-Te ECT-A.

\begin{tabular}{llllllll}
$*$ & * $p \leq 0,05 ;{ }^{* *} p \leq 0,01$. & Modalidade & \multicolumn{6}{c}{ Dimensóes } \\
\cline { 2 - 9 } & & TF & TT & EO & PM & RPN & RPP \\
\cline { 2 - 8 } & Coletiva & $0,32^{* *}$ & 0,10 & 0,14 & 0,00 & 0,00 & $0,18^{*}$ \\
& Individual & 0,19 & 0,00 & 0,18 & 0,12 & 0,13 & $0,25^{*}$ \\
\hline
\end{tabular}

$\mathrm{Na}$ comparação das respostas envolvendo todos os atletas e treinadores componentes da amostra - de acordo com a natureza coletiva ou individual de cada
Tendo em vista que os questionários possuem nível ordinal de mensuração e diferentes escalas, adotou-se a correlação não-paramétrica de Spearman (vide BARBETTA, 2004; TRIOLA, 1999). Este procedimento estatístico possibilitou verificar a significância de associação entre as percepçóes dos treinadores e dos seus atletas para cada uma das seis dimensōes avaliadas pelos testes. A fim de responder a outras questōes decorrentes das características dos sujeitos e dos resultados encontrados, também foram utilizados o Teste-t de Student e o Teste de Aderência do Qui-Quadrado.

esporte - observou-se baixos valores de correlação positiva e significativa $(\mathrm{p} \leq 0,05)$ envolvendo a percepção de ambos os grupos na dimensão RPN. Para treinadores e atletas de esportes coletivos também houve associação significativa, fraca e positiva na dimensão TF. Nessa mesma dimensão, não houve associação significativa para treinadores e atletas de modalidades individuais.

Nas demais dimensōes (TF; TT; EO; PM; RPP) - independentemente da natureza coletiva ou individual de cada esporte - foram observadas correlações fracas e não significativas entre as percepções dos atletas e de seus respectivos treinadores. Isso indica que as percepções de atletas e treinadores não estão associadas nestas importantes dimensões do comportamento do treinador. Em suma, na maioria das dimensões analisadas o comportamento declarado pelos treinadores foi parcial e limitadamente confirmado pelos atletas.

No entanto, as percepções de atletas e treinadores, de ambas as modalidades, sobre as mesmas questôes referentes a seis dimensōes do comportamento do treinador podem ter apresentado resultados tão dessemelhantes porque os treinadores se superestimaram. Eles podem, por exemplo, na questão: "Certifico-me de que o atleta esteja entendendo as técnicas e as estratégias que lhe estão sendo ensinadas?" terem assinalado a opção 5 (sempre) enquanto, de fato, não o fazem.

A fim de testar essa hipótese, oriunda dos resultados do presente estudo, calculou-se a razão das respostas dos treinadores em cada uma das questóes dentro das seis dimensões sob análise. Por exemplo, para uma dada questão da dimensão TF, a resposta do treinador foi divida por cinco: logo, se um treinador assinalou a opção 3 (metade das vezes) o resultado da razão foi 0,6 . O mesmo foi feito com as respostas dos atletas. Todavia, como 
a ECT-A oferece sete possibilidades de resposta o denominador, ao invés de cinco, foi sete.

Esse procedimento transformou uma escala categórica ordinal em quantitativa contínua variando de 0,2 a 1 para os treinadores e de 0,14 a 1 para os atletas. De posse destes resultados calculou-se a média das respostas de cada dimensão para atletas e treinadores de ambas as modalidades esportivas e, por fim, testou-se a hipótese nula de que os treinadores não se avaliam de forma mais positiva do que os atletas os avaliam. É importante frisar que o valor absoluto dos treinadores nunca foi inferior ao valor da razão dos atletas; logo não é plausível traçar hipóteses sobre aspectos em que os treinadores se subestimaram.

A FIGURA 1 apresenta os resultados para os sujeitos dos esportes coletivos e a FIGURA 2 para os sujeitos dos esportes individuais. É possível visualizar que os treinadores de esportes coletivos tenderam a supervalorizar suas qualidades comportamentais em cinco dimensōes (TT; PM; EO; RPP e RPN). A hipótese testada só não foi confirmada na dimensão TF. Por sinal, conforme análise precedente, essa dimensão foi a que apresentou maior compatibilidade entre as declaraçôes dos treinadores e as afirmaçôes dos atletas de esportes coletivos $(r=0,32)$.

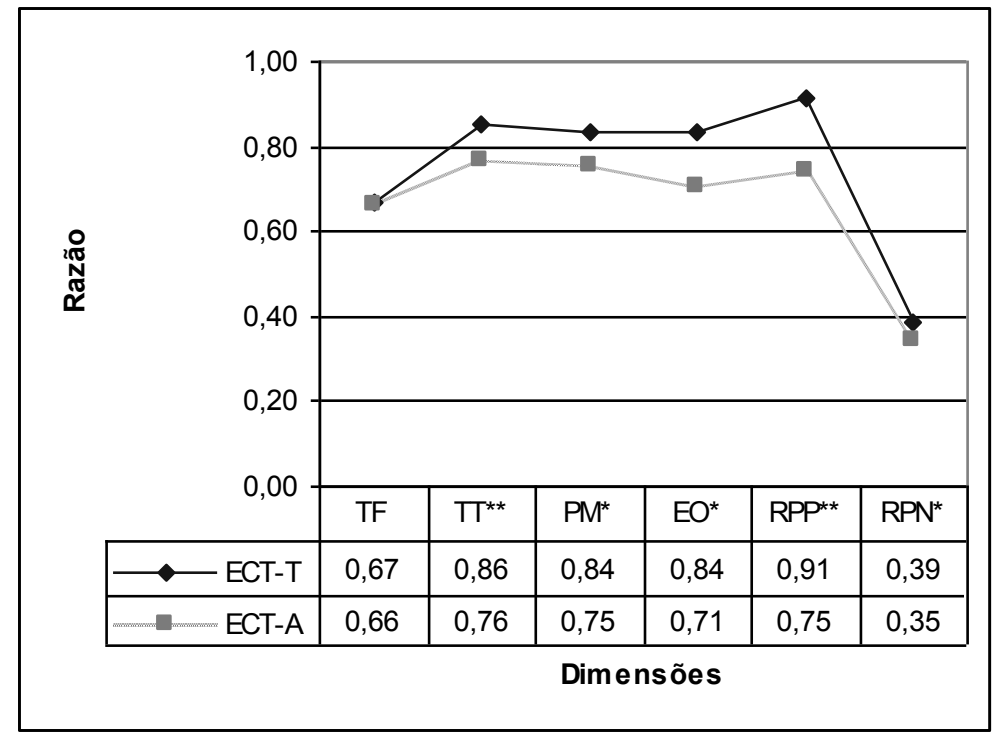

FIGURA 1 - Média da razão das respostas de treinadores e atletas de esportes coletivos em cada uma das seis dimensões dos questionários ECT-T e ECT-A.

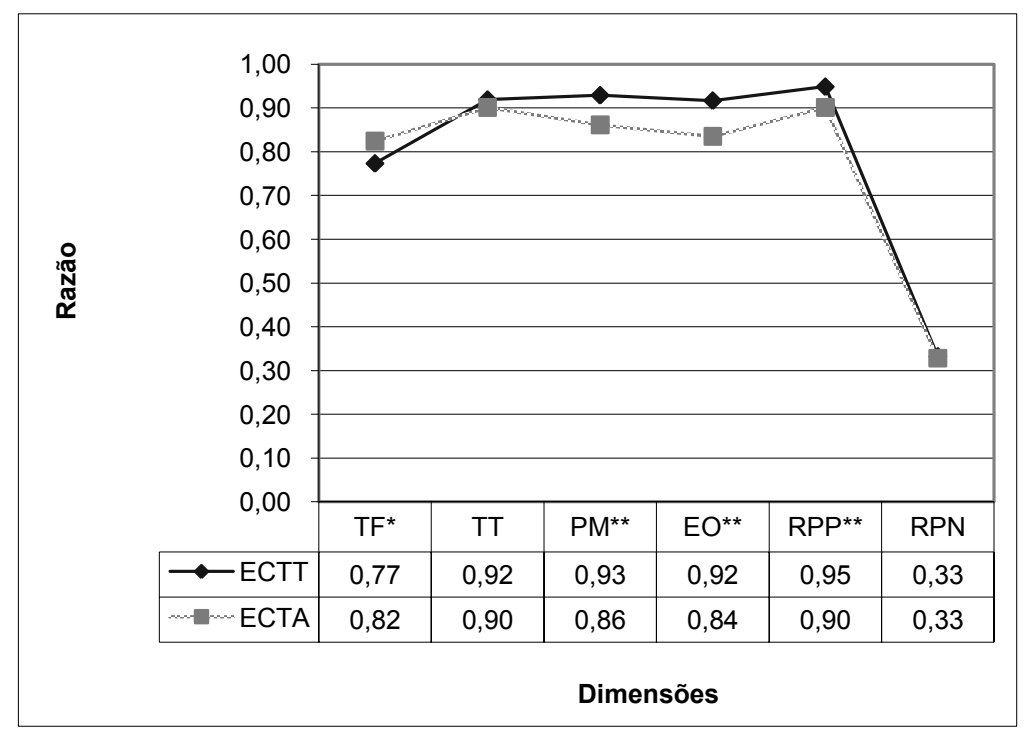

FIGURA 2 - Média da razão das respostas de treinadores e atletas de esportes individuais em cada uma das seis dimensões dos questionários ECT-T e ECT-A. 
Nos esportes individuais a hipótese nula não foi aceita em quatro dimensões: TF, PM, EO e RPP, sugerindo que os treinadores de esportes individuais também se superestimaram, o que provavelmente contribuiu para a não observância de correlação nessas dimensões e na dimensão TT.

Registrada a questão da supervalorização na resposta dos treinadores, ainda é possível, dados os resultados obtidos, sinalizar outra direção para a baixa compatibilidade das percepções de atletas de modalidades coletivas e individuais. Talvez o mais importante não seja investigar a questão da percepção de treinadores e atletas de acordo com o tipo de modalidade esportiva e sim, estudar as interações singulares entre um treinador e seus atletas.

Mediante essa inferência apresenta-se uma última tabela (TABELA 3). Nela é possível vislumbrar os coeficientes de correlação para cada conjunto de atletas e seus respectivos treinadores. Embora correlações estatisticamente significativas sejam raras, principalmente em decorrência da organização da amostra em grupos consideravelmente menores, é possível observar coeficientes de correlação baixos, moderados, altos, positivos e negativos. Por exemplo, na equipe $\mathrm{C} 7$, constatou-se uma correlação significativa positiva e forte $(\mathrm{p} \leq 0,01 ; \mathrm{r}=0,89)$ na dimensão EO; enquanto, nessa mesma dimensão, treinadores e atletas da equipe $\mathrm{C} 17$ parecem não se entender muito bem $(p \leq 0,01 ; r=-0,84)$. Casos semelhantes a esse podem ser vistos para diferentes treinadores e atletas de modalidades individuais.

Observam-se ainda casos de correlação positiva perfeita (I5 na dimensão RPP), e outros de correlação negativa quase perfeita (C19 na dimensão EO). Nota-se, ainda, que um mesmo treinador pode ter suas percepçóes forte e positivamente correlacionadas com a dos seus atletas em uma dimensão, enquanto em outra, a correlação é forte e negativa (C4 nas dimensões TF e PM). Estes resultados são discutidos a seguir, assim como os resultados para cada dimensão do comportamento do treinador acessados pela ECT-T e pela ECT-A.

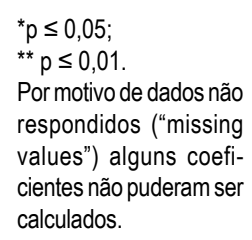
participantes do estudo.

\begin{tabular}{|c|c|c|c|c|c|c|c|}
\hline \multirow{2}{*}{ Modalidade } & \multirow{2}{*}{ Equipe } & \multicolumn{6}{|c|}{ Dimensões } \\
\hline & & TF & TT & EO & PM & RPP & RPN \\
\hline \multirow{19}{*}{$\frac{\tilde{z}}{0}$} & $\mathrm{C} 1$ & 0,41 & 0,22 & $-0,29$ & $-0,58$ & $-0,25$ & 0,60 \\
\hline & $\mathrm{C} 2$ & $-0,58$ & $-0,90$ & $-0,36$ & 0,87 & $-0,27$ & 0,24 \\
\hline & C3 & $1,00^{* *}$ & $1,00^{* *}$ & $1,00^{* *}$ & $1,00^{* *}$ & $1,00^{* *}$ & $1,00^{* *}$ \\
\hline & $\mathrm{C} 4$ & $0,90^{* *}$ & $-0,33$ & $-0,15$ & $-0,90^{*}$ & 0,66 & 0,62 \\
\hline & C5 & 0,36 & 0,23 & $-0,14$ & 0,45 & - & $-0,30$ \\
\hline & C6 & 0,26 & 0,15 & 0,00 & 0,71 & $0,84^{*}$ & 0,64 \\
\hline & $\mathrm{C} 7$ & 0,60 & $-0,46$ & $0,89^{*}$ & 0,00 & $-0,20$ & $-0,14$ \\
\hline & $\mathrm{C} 8$ & 0,43 & 0,49 & 0,41 & 0,00 & - & 0,34 \\
\hline & C9 & 0,15 & $-0,29$ & 0,71 & $-0,36$ & - & $-0,12$ \\
\hline & $\mathrm{C} 10$ & 0,14 & 0,04 & - & 0,74 & - & 0,10 \\
\hline & $\mathrm{C} 11$ & 0,50 & 0,00 & $-0,91^{*}$ & 0,30 & 0,64 & 0,10 \\
\hline & $\mathrm{C} 12$ & 0,32 & $-0,44$ & - & $0,89^{*}$ & 0,53 & 0,14 \\
\hline & $\mathrm{C} 13$ & 0,32 & - & 0,00 & $-0,35$ & - & 0,41 \\
\hline & $\mathrm{C} 14$ & $-0,26$ & $-0,24$ & $-0,65$ & 0,53 & $-0,41$ & 0,32 \\
\hline & $\mathrm{C} 15$ & 0,14 & $0,78^{*}$ & $-0,56$ & $-0,11$ & - & 0,62 \\
\hline & $\mathrm{C} 16$ & 0,40 & $-0,51$ & 0,87 & 0,00 & - & $-0,36$ \\
\hline & $\mathrm{C} 17$ & $-0,076$ & $-0,84^{* *}$ & 0,25 & $-0,41$ & 0,74 & $-0,88^{* *}$ \\
\hline & $\mathrm{C} 18$ & 0,50 & 0,41 & $-0,88^{*}$ & 0,22 & 0,72 & $-0,67$ \\
\hline & C19 & 0,49 & 0,45 & $-0,97^{* *}$ & $-0,35$ & 0,00 & 0,51 \\
\hline
\end{tabular}

Continua 
TABELA 3 - Coeficiente de correlação de Spearman sobre a percepção dos atletas de cada um dos 32 treinadores participantes do estudo (continuação).

\begin{tabular}{|c|c|c|c|c|c|c|c|}
\hline \multirow{2}{*}{ Modalidade } & \multirow{2}{*}{ Equipe } & \multicolumn{6}{|c|}{ Dimensóes } \\
\hline & & TF & TT & EO & PM & RPP & RPN \\
\hline \multirow{13}{*}{ 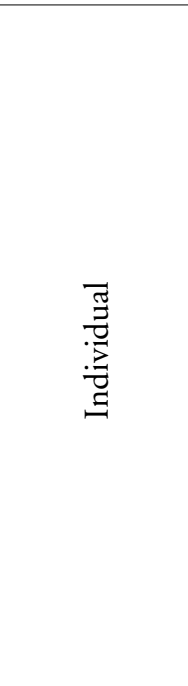 } & I1 & $-0,43$ & $-0,58$ & 0,00 & $-0,56$ & $-0,27$ & 0,69 \\
\hline & I2 & 0,20 & $-0,44$ & $-0,41$ & - & - & 0,48 \\
\hline & $\mathrm{I} 3$ & 0,39 & 0,38 & 0,79 & 0,40 & 0,00 & $0,94^{* *}$ \\
\hline & I4 & 0,26 & $0,76^{*}$ & - & - & - & - \\
\hline & I5 & 0,45 & - & - & - & - & $1,00^{* *}$ \\
\hline & I6 & $0,97^{* *}$ & $1,00^{* *}$ & $-0,61$ & $-0,61$ & $-0,61$ & 0,72 \\
\hline & I7 & 0,32 & $-0,55$ & - & 0,30 & - & 0,44 \\
\hline & I8 & 0,54 & 0,29 & - & 0,61 & - & 0,50 \\
\hline & I9 & $-0,38$ & 0,21 & $-0,25$ & 0,67 & $-0,20$ & $-0,10$ \\
\hline & I10 & 0,28 & - & - & - & - & - \\
\hline & I11 & 0,73 & 0,00 & - & 0,00 & $-0,32$ & $-0,10$ \\
\hline & $\mathrm{I} 12$ & 0,53 & $-0,22$ & - & - & 0,63 & - \\
\hline & I13 & 0,42 & $-0,20$ & $-1,00^{* *}$ & 0,61 & - & $-0,69$ \\
\hline
\end{tabular}

\section{Discussão}

\section{Dimensão TF}

Conforme apresentado, nessa dimensão observou-se uma correlação fraca e significativa (vide BARbetTA, 2004; Triola, 1999) entre treinadores e atletas de esportes coletivos. Tal resultado indica que a organização do treinamento, no quesito condicionamento físico, é raramente confirmada talvez por deficiências no planejamento do mesmo (processos de organização, treinamento e competição) ou por problemas no processo de comunicação envolvendo treinadores e atletas.

Essa fraca correspondência é um fator de preocupação para a maioria dos treinadores, pois a preparação física de qualidade é decisiva no comportamento atlético, conforme documentam Janelle e Hillman (2003). De fato, independentemente da origem do problema (organização do treinamento ou falhas no processo de comunicação), a baixa compatibilidade envolvendo as percepções do comportamento do treinador prejudica o desempenho esportivo e o desenvolvimento dos atletas conforme relata a literatura sobre o assunto (Chelladurai, 1990; Côté et al., 1995; Salmela, Young \& Kallio, 2000; SMITH \& SMOLL, 1984).

Entre os sujeitos das modalidades individuais não foi constatada correlação estatisticamente significativa. $\mathrm{O}$ resultado sugere que treinadores e atletas de desportos individuais percebem o treinamento físico de forma diferente. Essa diferença pode prejudicar o desenvolvimento de capacidades físicas dos atletas e, consequentemente, afetar negativamente a performance durante competições. Além disto, a baixa compatibilidade entre as percepçóes dos treinadores e atletas de desportos individuais na dimensão TF sugere pequena confiança do grupo de atletas nos itens referentes ao treinamento físico.

\section{Dimensão TT}

Nesta dimensão não existe correlação $(\mathrm{p}>0,05)$ entre as declarações dos treinadores e as respostas dos atletas, ou seja, em ambas as modalidades esportivas, há grande discordância entre treinadores e atletas.

Problemas de comunicação podem explicar essa pequena taxa de associação entre os líderes e os liderados. Pode existir, outrossim, uma lacuna na organização do treinamento. Semelhantemente ao apresentado nos fatores do treinamento físico, a organização do treinamento técnico pode não ser tão bem elaborada e, por este motivo, os atletas têm dificuldades de assimilar e interagir com os comportamentos adotados por seus treinadores nesta dimensão do treinamento esportivo. Especificamente para o problema da compatibilidade das percepções dos atletas e dos seus treinadores na dimensão treinamento técnico,
${ }^{*} p \leq 0,05$

${ }^{* *} p \leq 0,01$.

Por motivo de dados não respondidos ("missing values") alguns coeficientes não puderam ser calculados. 
algumas medidas poderiam ser tomadas a fim de aprimorar este aspecto da preparação dos atletas.

Dentre essas medidas, destaca-se a reavaliação dos processos de organização, treinamento e competição, pois uma estrutura de treinamento bem elaborada requer uma congruência entre o planejado e o treinado, o que aumenta a possibilidade de rendimento esportivo, conforme sugerido por vários estudiosos (CÔTÉ et al., 1995; ErICSSON, 2003).

\section{Dimensão PM}

Novamente, tendo em vista os coeficientes de correlação e o valor $p$ superior a 0,05 , constatou-se que as declarações de treinadores e atletas - tanto os de esportes coletivos quanto os de individuais - mostraram-se independentes. Portanto, o que os treinadores afirmam não é confirmado pelos atletas.

Diante dessa constatação, pode-se afirmar que haveria necessidade de um programa de habilidades mentais, administrado pelo próprio treinador ou por um especialista em psicologia do esporte, que enfatizasse, por exemplo, o aprimoramento da capacidade de controlar a ansiedade e, em esportes coletivos, o desenvolvimento da coesão do grupo.

A adoção de um programa efetivo de ensino de habilidades mentais compreende o controle da ansiedade, da ativação, da capacidade de focalização e de outras habilidades que podem ser aprimoradas por meio do treinamento psicológico e a partir do estabelecimento de condiçóes ideais de aprendizagem, conforme amplo respaldo literário (DuRAND-Bush; SALMELA \& GREENDemers, 2001; Moraes \& LÔbo, 2001; OrLick, 2000).

\section{Dimensão EO}

Para as questôes referentes à dimensão $\mathrm{EO}$ não foram constatadas diferenças estatisticamente significativas ( $\mathrm{p}$ $>0,05)$ para as respostas dos treinadores e dos atletas, comprovando que, nesta dimensão, independente do tipo de modalidade (coletiva ou individual), inexiste associação entre o conteúdo das respostas dos treinadores com o de seus atletas.

Possivelmente, os treinadores avaliados não apresentam um programa específico de estabelecimento de objetivos para os seus atletas. A carência deste tipo de programa pode alterar a eficiência da liderança do treinador, visto que a definição de liderança perpassa pelo estabelecimento de metas: "processo comportamental de influenciar indivíduos e grupos na direção de metas estabelecidas" (WeInBerg \& Gould, 2001). Seria interessante que o treinador obtivesse informações que o auxiliassem no processo de estabelecimento de metas: uma consultoria com profissional especializado na área, como um preparador mental, por exemplo.

Incompatibilidades envolvendo as características pessoais de treinadores e atletas também podem estar associadas a este resultado pois, conforme descrito na literatura (WEINBERG \& GoULD, 2001), treinadores e atletas que possuem filosofias e níveis de aspiração muito diferentes têm poucas chances de serem bem sucedidos em um programa de estabelecimento de metas.

\section{Dimensão RPP}

Dada a significância dos coeficientes de correlação, pode-se afirmar que, nesse fator do treinamento esportivo, as percepçōes dos treinadores e atletas de esportes coletivos e individuais são independentes. Há necessidade de se dispensar ampla atenção a esse resultado.

Afinal, o reforço pessoal positivo é fundamental para a superação de restriçôes motivacionais de naturezas diversas e a afinação dos processos de comunicação entre os treinadores e os seus atletas é imprescindível para aumentar a coesão e o rendimento tanto em esportes coletivos quanto em modalidades individuais.

De fato, conforme descreve a literatura (Chelladurai, 1984; Cótê et al., 1999; LOBo; Moraes \& NASCIMENTO, 2005; Silveira, 2005), a comunicação efetiva e a interação positiva entre líderes e liderados estão associadas ao aprimoramento da performance.

\section{Dimensão RPN}

Em relação às questões dessa dimensão, observouse diferença estatisticamente significativa ( $\mathrm{p} \leq$ $0,05)$ para as respostas dos treinadores e dos atletas das modalidades coletivas e individuais. Nesta variável do treinamento esportivo, existe associação significativa entre as percepções de treinadores e seus respectivos atletas. Além disto, dados os valores absolutos dos coeficientes de correlação, há uma maior compatibilidade entre treinadores e atletas de modalidades individuais.

Esse resultado pode refletir uma maior interação entre eles visto que, em modalidades individuais, ao treinar um menor número de atletas, há mais possibilidades e tempo para os treinadores conhecerem características comportamentais e reaçōes emocionais de seus atletas.

A possível existência de uma relação mais próxima entre treinadores e atletas de modalidades individuais pode fazer com que os primeiros forneçam menor frequência de reforço pessoal negativo aos segundos. Todavia, novamente reportando aos 
coeficientes de correlação, deve-se ressaltar que a força da associação entre treinadores e atletas é fraca, independentemente do tipo de modalidade. Em suma, treinadores e atletas apresentam opinióes pouco semelhantes e é necessário o aprimoramento do grau de similaridade das percepçóes entre eles nessa dimensão do comportamento do treinador.

Afinal, caso o treinador tenha pouca percepção, que cria, eventualmente, um ambiente desfavorável de aprendizagem e treinamento - marcado por críticas e repreensões aos atletas - ele pode aumentar a probabilidade de sentimentos de insatisfação, ansiedade e incompetência em seu grupo de atletas; aumentando também a chance de abandono do esporte, "burnout", por eles.

Ainda sobre esta temática, ressalta-se que a importância do estabelecimento de um ambiente favorável de aprendizagem, onde as críticas sejam reduzidas e construtivas e os atletas sejam apoiados é descrita por vários autores (CHELladduRa, 1990; Moraes \& Salmela, 2002; Salmela, 1996; Weinberg \& Gould, 2001).

Recapitulando, verificou-se que as percepçōes dos treinadores e dos atletas apresentaram pouca similaridade

\section{Conclusões}

Conclui-se que inexiste correlação entre as percepções de atletas e treinadores de modalidades coletivas em quatro dimensóes do comportamento do treinador (TT; EO; PM; RPP). Entre treinadores e atletas de modalidades individuais também não foram verificadas associações significativas ao nível de $\alpha=$ 0,05 em cinco dimensões (TF; TT; EO; PM; RPP).

Nas dimensões que apresentaram significância estatística (TF e RPN para esportes coletivos; RPN para esportes individuais) os coeficientes de correlação foram baixos, de modo que o comportamento relatado pelos treinadores foi parcial e limitadamente confirmado pelos atletas. A baixa compatibilidade entre as percepções de atletas e treinadores está relacionada a falhas no processo de organização do treinamento, no processo de comunicação, além de incompatibilidade entre as características pessoais dos treinadores e desportistas.

Sendo a percepção dos atletas pouco análoga à dos treinadores, sugerem-se futuras intervençōes para melhora dos processos de interação treinador-atleta e, consequentemente, para o aumento da satisfação do grupo de desportistas e aprimoramento da performance competitiva. Afinal, tendo em vista que os treinadores em todas as seis dimensōes do comportamento do treinador analisadas. Notou-se, inclusive, a inexistência de qualquer tipo de associação nas dimensões TT; PM; EO eRPP para atletas e treinadores de modalidades coletivas, assim como nas dimensões TF, TT, PM, EO e RPP para os sujeitos do grupo individual. Como abordado anteriormente, treinadores e atletas apresentaram opiniōes pouco semelhantes devido à provável existência de falhas no processo de organização do treinamento e competição, no processo de comunicação, por incompatibilidade entre as características pessoais dos líderes e dos atletas, dentre outros motivos.

Finalmente, a existência de grande variabilidade na interação de treinadores e atletas sugere que, no que concerne ao estudo dos processos de interação envolvendo treinadores e atletas, uma abordagem idiográfica - "método de conhecimento científico que trata de fatos considerados individualmente" (Houaiss, Villar \& Franco, 2001) - pode ser mais elucidativa do que uma nomotética - "método que formula ou trata de leis gerais para o entendimento de um determinado evento, circunstância ou objeto" (Houaiss, Villar \& Franco, 2001).

são considerados cruciais para o desenvolvimento pessoal e esportivo dos atletas, a fraca associação entre o conteúdo das respostas destes com o de seus atletas, em várias dimensões do comportamento do treinador, pode resultar em queda de desempenho ou na estagnação do desenvolvimento do potencial dos atletas.

Além do mais, em várias dimensōes da ECT-T (TT; PM; EO; RPP e RPP para treinadores de esportes coletivos; TF; PM; EO e RPP para os treinadores de esportes individuais), os treinadores se avaliaram de forma mais positiva (para $\alpha=0,05$ ) do que os atletas os avaliaram. Logo, o fato dos atletas raramente confirmarem a percepção dos seus treinadores, também reflete uma auto-valorização excessiva em relação à qualidade dos seus comportamentos.

A hipótese de que o nível de associação, dado pelo valor absoluto do coeficiente de correlaçáo entre as percepçôes de atletas e treinadores de esportes individuais, seria maior do que a envolvendo treinadores e atletas de modalidades coletivas só foi confirmada na dimensão RPP. Na dimensão TF ocorreu o contrário e, nas demais dimensōes, conforme supracitado, não foram verificadas associaçôes estatisticamente significativas entre as declarações de treinadores e atletas. Estes resultados 
não sofreram influências do tempo de convivência envolvendo treinadores e atletas dos dois grupos, assim como do nível de instrução formal dos treinadores.

Verificou-se ainda que o relacionamento singular envolvendo cada treinador e os atletas sob o seu comando informa mais do que a simples classificação: treinadores e atletas de esportes coletivos; treinadores e atletas de esportes individuais.

Por fim, a utilização conjunta dos questionários ECT-T e ECT-A mostrou-se valiosa opção para pesquisadores interessados em estudar o processo de interação treinadores-atletas. Dentre outras possibilidades, futuras pesquisas utilizando estes instrumentos poderiam focalizar no estudo de treinadoras, atletas de outros níveis de desempenho (não estaduais, como no presente estudo), na influência do tempo de convivência, na compatibilidade entre as percepçôes de treinadores e atletas e na verificação da percepção de titulares e reservas em relação a um mesmo treinador (a) de um desporto coletivo.

\begin{abstract}
Scale of coach behavior - version coach (ECT-T) and version athlete (ECT-A): what the coach says is confirmed by its athletes?
\end{abstract}

The purpose of this study was to verify the level of association between athletes and their coaches' perceptions, according to the kind of sport (individual or group), in the six dimensions of coaching behavior - Physical Training (PT); Technical Training (T); Mental Preparation (MP); Goal Setting (GS); Personal Positive Rapport (PPR) Personal Negative Rapport (PNR) - accessed by the two Brazilian versions of Coaching Behavior Scale for Sport: Coaches (ECT-T) and Athletes Versions (ECT-A). Participated 181 athletes ( 114 male and 67 female) of nine different sports with mean age of 17.15 years $( \pm 1.48)$ and their respective coaches $(N=32)$, all male, with mean age of 32.88 years $( \pm 8.91)$. Coaches and athletes answered the 38 items, which have the same content, in the mentioned questionnaires. The non-parametric Spearman correlation showed the inexistence of significant association, at $\alpha$ level $=0.05$, between the coaches and athletes perception of group sports in the dimensions $\Pi, M P, G S$ and PPR. In addition, there were no significant correlations between coaches and athletes perceptions of individual sports in the following dimensions: PT, $\Pi, M P, G S$ and PPR. Among others reasons, the low compatibility involving the coaches and athletes perceptions can be associated with failures in the training organization process. Besides, the relationship of each coach with their athletes appears to add more information than the simple categorization: group modalities versus individual modalities.

UnITERMS: Coaches' behaviors; Leadership; Sport psychology; ECT-T \& ECT-A.

\title{
Referências
}

BARBETTA, P.A. Estatística aplicada às ciências sociais. 5. ed. Florianópolis: Universidade Federal de Santa Catarina, 2004. BLOOM, B.S. Developing talent in young people. New York: Ballentine, 1985.

CHELlADURAI, P. Leadership in sport. In: SILVA, J.M; WEINBERG, R. S. (Org.). Psychological foundations of sport. Champaign: Human Kinetics. 1984. p.329-39.

Leadership in sport: a review. International Journal of Sport Psychology, Rome, v.21, n.4, p.329-54, 1990.

CÔTÉ, J.; SALMELA, J.H.; TRUDEL, P.; BARIA, A.; RUSSELL, S.J. The coaching model: a grounded assessment of expert gymnastic coaches' knowledge. Journal of Sport and Exercise Psychology, Champaign, v.17, p.1-17, 1995. CÔTÉ, J.; YARDLEY, J.; HAY, J.; SEDGWICK, W.; BAKER, J. An exploratory examination of the coaching behavior scale for sport. Avante Research Note, v.5, n.2, p.82-92, 1999.

DURAND-BUSH, N.; SALMELA, J.H.; GREEN-DEMERS, I. The ottawa mental skills assessment tool (omsat3*). The Sport Psychologist, Champaign, v.15, p.1-19, 2001.

ERICSSON, K.A. Development of elite performance and deliberate practice: an update from the perspective of the expert performance approach. In: STARKES, J.L.; ERICSSON, A.K. (Orgs.). Expert performance in sports. Champaign: Human Kinetics, 2003. p 49-83. 
ERICSSON, K.A.; KRAMPE, R.; TESCH-RÖMER, C. The role of deliberate practice in the acquisition of expert performance. Psychological Review, Washington, v.3, n.100, p.363-406, 1993.

JANELLE, C.M.; HILLMAN, C.H. Expert performance in sport: current perspectives and critical issues. In: STARKES, J.L.; ERICSSON, A.K. (Orgs.). Expert performance in sports. Champaign: Human Kinetics, 2003.

HOUAISS, A.; VILLAR, M.S.; FRANCO, M.M. Dicionário Houaiss da língua portuguesa. Rio de Janeiro: Objetiva, 2001. LÔBO, I.L. MORAES; L.C.; NASCIMENTO, E. Processo de validação da escala de comportamento de treinadores: versão atleta (ECT-A). Revista Brasileira de Educação Física e Esporte, São Paulo, v.19, n.3, p.255-65, 2005.

MEDEIROS FILHO, E.S.; MORAES, L.C. Perfil de Treinadores brasileiros experts de judô. In: CONGRESSO BRASILEIRO E INTERNACIONAL DE PSICOLOGIA DO ESPORTE, 1., 2004, Curitiba. Anais... Curitiba: Sociedade Brasileira de Psicologia do Esporte, 2004.

MORAES, L.C. Looking back \& thinking ahead: reflections and recommendations. In: SALMELA, J.H. (Org). Great job coach! Getting the edge from proven winners. Ottawa: Potentium, 1996. p.207-29.

MORAES, L.C.; LÔBO, I.L. Análise da ansiedade pré-competitiva de atletas de diferentes esportes. In: GARCIA, E.S; MORAES, L.C. Olimpíada Colegial dos Jogos da Esperança 2001 Poços de Caldas - MG: resultados. Belo Horizonte: Ministério do Esporte e Turimo, 2002.

MORAES, L.C.; SALMELA, J.H. Expertise no esporte: considerações contextuais. In: SILAMI, E.G; LEMOS, K.L.

Temas atuais em educação física e esportes VII. Belo Horizonte: Health, 2002. cap.11, p.159-72.

ORLICK, T. In pursuit of excellence: how to win in sports and life through mental training. 3rd. ed. Champaign: Human Kinetics, 2000.

SALMELA, J.H. Expert coaches strategies for the development of expert athletes. In: ROGOZKIN, R.J.; MAUGHAN, M.(Orgs). Current research in sports sciences. New York: Plenum Press, 1996.

SALMELA, J.H.; MORAES, L.C. Development of expertise: the role of coaching, families and cultural contexts. In. STARKES, J.L.; ERICSSON, A.K. (Orgs). Expert performance in sports. Champaign: Human Kinetics, 2003. p.272-91. SALMELA, J.H.; YOUNG, B.W.; KALLIO, J. Within-career transitions of the athlete-coach-parent triad. In: LAVALLEE. D.; WYLLEMAN, P. Career transitions in sport: international perspectives. Champaign: Human Kinetics, 2000. p.181-93. SILVEIRA, D.R. Processo de validação da escala de comportamento de treinadores: versão treinador (ECT-T). 2005. 128 f. Dissertação (Mestrado em Treinamento Esportivo) - Escola de Educação Física, Fisioterapia e Terapia Ocupacional, Universidade Federal de Minas Gerais, Belo Horizonte, 2005.

SMITH, R.E; SMOLL, F.L. Leadership research in youth sports. In: SILVA III, J.M.; WEINBERG, R.S. (Orgs.). Psychological foundations of sport. Champaign: Human Kinetics, 1984. p.371-86.

TRIOLA, M.F. Introdução à estatística. 7. ed. Rio de Janeiro: LTC, 1999.

WEINBERG, R.S.; GOULD, D. Fundamentos da psicologia do esporte e do exercício. 2. ed. Porto Alegre: Artmed, 2001.

\section{Agradecimentos}

Os autores agradecem a Fundação de Amparo à Pesquisa do Estado de Minas Gerais (FAPEMIG) pelo auxílio financeiro concedido no processo de desenvolvimento deste trabalho.

\begin{tabular}{r|l} 
ENDEREÇo & \\
Luiz Carlos Moraes & \\
CENESP/UFMG & Recebido para publicação: 19/04/2007 \\
Av. Pres. Carlos Luz, 6627 & 1a. Revisão: 26/05/2008 \\
e-mail: lmoraes@ufmg.br & 2a. Revisão: 10/o8/2009 \\
& 3a. Revisão: 13/08/2009 \\
& Aceito: 23/09/2009
\end{tabular}

